\title{
UV Dose Governs UV-Polymerized Polyacrylamide Hydrogel Modulus
}

\author{
Saahil Sheth, Era Jain, Amin Karadaghy, Sana Syed, \\ Hunter Stevenson, and Silviya P. Zustiak \\ Department of Biomedical Engineering, Parks College of Engineering, Aviation and Technology, \\ Saint Louis University, 3507 Lindell Blvd., St. Louis, MO 63103, USA \\ Correspondence should be addressed to Silviya P. Zustiak; szustiak@slu.edu
}

Received 19 April 2017; Accepted 24 August 2017; Published 10 October 2017

Academic Editor: Shida Miao

Copyright (C) 2017 Saahil Sheth et al. This is an open access article distributed under the Creative Commons Attribution License, which permits unrestricted use, distribution, and reproduction in any medium, provided the original work is properly cited.

Polyacrylamide (PAA) hydrogels have become a widely used tool whose easily tunable mechanical properties, biocompatibility, thermostability, and chemical inertness make them invaluable in many biological applications, such as cell mechanosensitivity studies. Currently, preparation of PAA gels involves mixtures of acrylamide, bisacrylamide, a source of free radicals, and a chemical stabilizer. This method, while generally well accepted, has its drawbacks: long polymerization times, unstable and toxic reagents, and tedious preparation. Alternatively, PAA gels could be made by free radical polymerization (FRP) using ultraviolet (UV) photopolymerization, a method which is quicker, less tedious, and less toxic. Here, we describe a simple strategy based on total UV energy for determining the optimal UV crosslinking conditions that lead to optimal hydrogel modulus.

\section{Introduction}

In recent years, polyacrylamide (PAA) hydrogels have gained popularity as substrates for cell culture due to their biocompatibility, hydrophilicity, low cost, and ease of preparation, as well as their attractive mechanical properties $[1,2]$. Another advantage of PAA hydrogels is the ability to vary the ratio of monomer (acrylamide) to crosslinker (bisacrylamide) and total monomer concentration; these strategies lead to hydrogels with Young's modulus anywhere between 0.1 and $300 \mathrm{kPa}$ and emulate any physiological soft tissue [3]. PAA hydrogels are commonly implemented as cell substrates to assess stiffness-dependent cell behavior due to their tunable compliance. For example, in the seminal paper by Lo et al. [4], the authors used PAA hydrogels of two different moduli to demonstrate that cells preferentially move from a softer substrate to a stiffer substrate, a phenomenon known as durotaxis. Since then, PAA hydrogels of different moduli have been used to demonstrate the role of substrate stiffness on cell morphology and the cytoskeleton [5], cancer cell metastasis and invasiveness $[6,7]$, cellular responses to drugs and toxins $[8,9]$, and directing cell phenotype [10].
Currently, when used as cell culture substrates, PAA gels are mainly polymerized with catalysts such as tetramethylenediamine (TEMED) and ammonium persulfate (APS), which are highly toxic and also lead to slow polymerization times-on the order of $45 \mathrm{~min}$ to $1 \mathrm{~h}$ for lower gel precursor concentrations $[1,11]$. Photocrosslinking with various photoinitiators, such as Irgacure 2959, has been more recently employed for the fabrication of PAA hydrogels with a stiffness gradient $[1,10,12]$, or for the quick preparation of large PAA hydrogel arrays for applications such as drug screening [8]. Photocrosslinking circumvents the use of toxic catalysts and is typically much faster-on the order of $1-5 \mathrm{~min}$ [8]. Final properties of UV-polymerized gels depend on the UV wavelength, uniformity, light intensity, and exposure times [1].

In this study, we present a method for preparing UVpolymerized PAA hydrogels with a focus on UV dose, rather than UV intensity or UV exposure time independently. We demonstrated that, for UV-polymerized hydrogels, rather than independently varying UV exposure time and intensity, one should consider the UV dose: interestingly, for all PAA hydrogels as well as UV parameter combinations tested here, 
TABLE 1: Concentrations and volumes of acrylamide and bisacrylamide required to prepare polyacrylamide hydrogels of various moduli.

\begin{tabular}{lccccc}
\hline Nomenclature & $\begin{array}{c}\text { Acrylamide } \\
\text { concentration (\%) }\end{array}$ & $\begin{array}{c}\text { Bisacrylamide } \\
\text { concentration (\%) }\end{array}$ & $\begin{array}{c}\text { Acrylamide from 40\% } \\
\text { stock solution (mL) }\end{array}$ & $\begin{array}{c}\text { Bisacrylamide from } \\
\text { stock solution } \\
(\mathrm{mL})\end{array}$ & $\begin{array}{c}\text { DI water (mL) } \\
\text { A5-B.025 }\end{array}$ \\
A5-B.10 & 5 & 0.025 & 0.625 & 0.063 & 4.31 \\
A8-B.10 & 5 & 0.100 & 0.625 & 0.250 & 4.13 \\
A8-B.25 & 8 & 0.100 & 1.000 & 0.250 & 3.75 \\
A12-B.25 & 12 & 0.250 & 1.000 & 0.00 & 0.625 \\
\hline
\end{tabular}

the optimal UV dose was $\sim 5 \times 10^{3} \mathrm{~mJ} / \mathrm{cm}^{2}$. The optimal dose was defined as the lowest dose required to reach hydrogel modulus saturation, above which the modulus did not change appreciably.

\section{Materials and Methods}

2.1. Materials. Acrylamide (A) (40\% w/v), bisacrylamide (B) $(2 \% \mathrm{w} / \mathrm{v})$, and Irgacure 2959 were purchased from BASF Corporation (Florham Park, NJ); phosphate-buffered saline (PBS) and Repel Silane ${ }^{\circledR}$ were purchased from GE Healthcare Life Sciences (Logan, UT); and silicone spacers were purchased from Grace Bio-Labs (Bend, OR). RPMI media and fetal bovine serum (FBS) were purchased from GE Healthcare Hyclone (Little Chalfont, UK). Trypsin was purchased from Sigma-Aldrich (St. Louis, MO). MDA-MB-231 breast cancer cells were obtained from NCI DCTD Repository (NCI, Frederick, MD). Type I collagen and sulfosuccinimidyl-6( $4^{\prime}$-azido-2' -nitrophenylamino)hexanoate (Sulfo-SANPAH) were purchased from Thermo Fisher (Waltham, MA), and penicillin-streptomycin (pen/strep) was purchased from MP Biomedicals (Santa Ana, CA).

\subsection{Hydrogel Preparation}

2.2.1. UV-Polymerized Hydrogels. To prepare the PAA gel precursor solution, A, B, and deionized (DI) water were added at specific ratios (Table 1) to prepare $5 \mathrm{~mL}$ working solutions. The gel precursor solution was degassed for $30 \mathrm{~min}$ at which point the photoinitiator, Irgacure 2959 for UV-crosslinked gels, was added to the solution at $0.1 \% \mathrm{w} / \mathrm{v}$ final concentration and mixed gently. Irgacure 2959 was chosen because it has been shown to be UV sensitive (at UV wavelength of $365 \mathrm{~nm}$ ) and also nontoxic to cells at low concentrations [13]. The solution was then pipetted on the center of a glass slide that was pretreated with Repel Silane to provide a hydrophobic surface. Silicone spacers $(0.5 \mathrm{~mm}$ thick $)$ were placed at the ends of the glass slide and a second hydrophobic-treated slide was placed on top; hence, the resulting uncured material had a uniform thickness of $0.5 \mathrm{~mm}$ to ensure uniform cure throughout the depth of the material. The slides were exposed to UV light (365 nm; IntelliRay 600W, Uvitron International, Inc.) of various intensities for various amounts of time to initiate crosslinking. Intensity was varied by placing the gels at a predetermined distance from the light source, where values for light intensity as a function of distance were measured and provided by the manufacturer.

2.2.2. Free Radical-Polymerized Hydrogels. TEMED-crosslinked gels were prepared in a similar way as described in the previous subsection with several notable differences. Briefly, to prepare $5 \mathrm{ml}$ of gel precursor solution, $\mathrm{A}, \mathrm{B}$, and deionized water were mixed at a desired ratio to give $4,972.5 \mu \mathrm{l}$ total volume. Upon degassing the precursor solution for $30 \mathrm{~min}$, $25 \mu \mathrm{l}$ APS (10\% w/v stock solution in deionized water; BioRad, Hercules, CA) and $2.5 \mu$ l of TEMED (Bio-Rad, Hercules, CA) were added. The solution was then again sandwiched between two hydrophobic-treated glass slides separated by spacers and left in a degassing chamber under vacuum for $45 \mathrm{~min}$ to polymerize.

2.3. Rheological Testing. Hydrogel slabs ( $0.5 \mathrm{~mm}$ thick) were swollen in PBS overnight and cut into circular slabs $20 \mathrm{~mm}$ in diameter for rheological testing. Residual buffer from the hydrogel surface was carefully blotted with a KimWipe ${ }^{\circledR}$ prior to measurement to avoid slipping. The storage modulus $\left(G^{\prime}\right)$ and loss modulus $\left(G^{\prime \prime}\right)$ were measured with an AR 2000ex rheometer (TA Instruments, New Castle, DE) in a parallel plate geometry with a $20 \mathrm{~mm}$ diameter, flat upper plate, at $22^{\circ} \mathrm{C}$, a frequency of $1-10 \mathrm{rad} / \mathrm{s}$, a constant $2 \%$ strain, and a normal force of $0.20-0.25 \mathrm{~N}$ [14]. Young's modulus was related to $G^{\prime}$ by the following equation:

$$
E=G^{\prime} 2(1+v)
$$

where $E$ is Young's modulus and $v$ is Poisson's ratio which was approximated to 0.5 for PAA gels $[15,16]$.

\subsection{Sample Preparation for Testing the Effect of \\ Polymerization Conditions on Hydrogel Stiffness}

2.4.1. Effect of UV Intensity. A8-B.10 and A12-B.25 hydrogel precursor solutions were prepared in $50 \mathrm{ml}$ conical vials. To degas the hydrogel precursor solution, the conical vials were left uncapped and placed in a degassing chamber under vacuum for $30 \mathrm{~min}$. Next, $350 \mu \mathrm{L}$ samples of each of the solutions were placed between two hydrophobic-treated glass slides. The slides were then placed in a UV oven with the following settings: exposure time of $300 \mathrm{~s}$ and/or $100 \mathrm{~s}$ and UV intensity of $15,26,67$, and $131 \mathrm{~mW} / \mathrm{cm}^{2}$. 


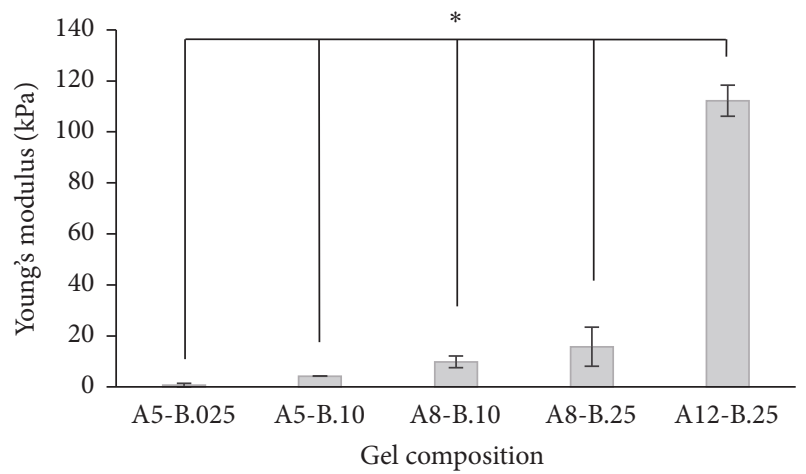

(a)

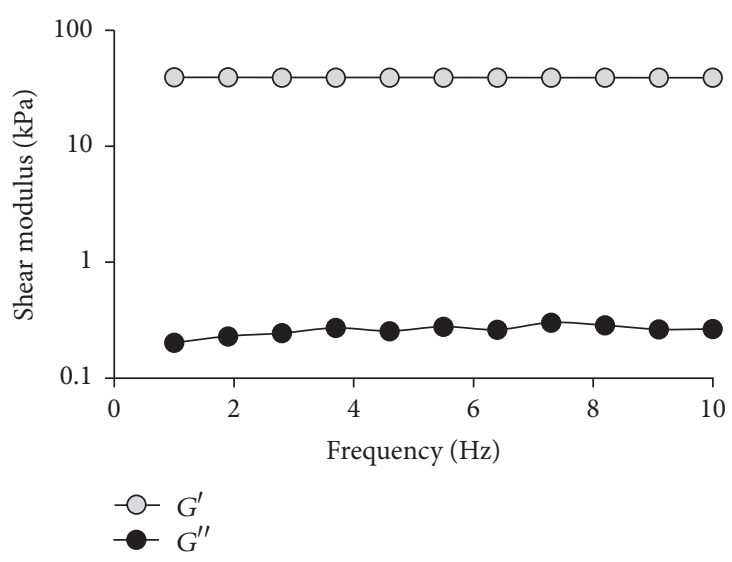

(b)

FIGURE 1: (a) Young's modulus as a function of gel composition for TEMED-crosslinked PAA hydrogels. (b) Representative shear stress as a function of frequency plot for A12-B0.25 PA hydrogel. Asterisks designate significant differences for $p<0.05, n=3$.

2.4.2. Effect of Exposure Time. A8-B.10 and A12-B.25 hydrogel precursor solutions were prepared in a $50 \mathrm{ml}$ conical vial and degassed for $30 \mathrm{~min}$, and $350 \mu \mathrm{L}$ samples of each were placed between two hydrophobic-treated glass slides. The slides were then placed in a UV oven with the following settings: exposure time of 50 s, 75 s, 100 s, 125 s, and $300 \mathrm{~s}$ and $\mathrm{UV}$ intensity of $15 \mathrm{~mW} / \mathrm{cm}^{2}$.

2.4.3. Effect of Irgacure Concentration. UV-crosslinked gels were prepared with various amounts of Irgacure 2959: 0.01\%, $0.10 \%$, and $0.50 \%$. Precursor solutions of A8-B.10 gels were prepared, degassed for $30 \mathrm{~min}$, and placed between two hydrophobic-treated glass slides. The slides were placed in the UV oven to polymerize at an intensity of $15 \mathrm{~mW} / \mathrm{cm}^{2}$ for $300 \mathrm{~s}$.

2.4.4. Effect of UV Dose. PAA hydrogels were fabricated at different doses (i.e., total energy) calculated as follows:

$$
\text { Dose }=\text { intensity } \times \text { time, }
$$

where the dose is in $\mathrm{mJ} / \mathrm{cm}^{2}$, intensity is in $\mathrm{mW} / \mathrm{cm}^{2}$, and time is in s. UV-crosslinked gels were prepared at various doses ranging from $\sim 1 \mathrm{~mJ} / \mathrm{cm}^{2}$ to $\sim 40 \mathrm{~mJ} / \mathrm{cm}^{2}$. Two types of PAA gels were prepared for this experiment to compare the effects of dose on gels with different moduli: a stiff gel (A12-B.25) and a soft gel (A8-B.10). For the stiff gel, three different UV intensities were used $\left(15,26\right.$, and $\left.67 \mathrm{~mW} / \mathrm{cm}^{2}\right)$, while the exposure time was steadily increased to obtain increasing dose values. For the soft gel, the intensity was held at $15 \mathrm{~mW} / \mathrm{cm}^{2}$, while the exposure time was again increased to obtain an increasing dose.

2.5. Collagen Coating of PAA Gels and Cell Culture. The PAA gels of desired moduli were prepared using UV-initiated polymerization. The PAA gels were coated with $0.1 \mathrm{mg} / \mathrm{mL}$ Type I collagen using a Sulfo-SANPAH crosslinker $(50 \mathrm{mg} / \mathrm{ml})$ as described by us previously [17]. Gel plates were then sterilized under UV light $(302 \mathrm{~nm})$ for $2 \mathrm{~h}$. The coated gels were then washed 2 times in PBS and equilibrated with $1 \mathrm{x}$ RPMI medium supplemented with $10 \% \mathrm{v} / \mathrm{v}$ FBS and $1 \% \mathrm{v} / \mathrm{v}$ pen/strep overnight. MDA-MB-231 human breast cancer cells were cultured on the hydrogels and maintained using regular cell culture techniques in RPMI medium supplemented with $10 \% \mathrm{v} / \mathrm{v}$ FBS and $1 \% \mathrm{v} / \mathrm{v}$ pen/strep. Cells were harvested through exposure to $0.5 \% \mathrm{v} / \mathrm{v}$ trypsin/EDTA for $5 \mathrm{~min}$ and seeded onto the PAA gels at a concentration of 1 $\times 10^{5}$ cells $/ \mathrm{cm}^{2}$. After supplementing with complete medium, cells were placed in a humidified incubator at $37^{\circ} \mathrm{C}$ and $5 \%$ $\mathrm{CO}_{2}$ and cultured for $24 \mathrm{~h}$.

2.6. Cell Imaging and Morphology. Cells were stained with acridine orange (green fluorescence, membrane permeable dye) at $24 \mathrm{~h}$ of culture on the PAA gels. Bright field and fluorescent images were captured using an inverted fluorescent microscope (Axiovert 200, Zeiss, Germany) with a 20x objective. Images were analyzed for cell spreading using the line plug-in on ImageJ (NIH free software).

2.7. Statistical Analysis. The results of all experiments are reported as the mean values $( \pm S D)$ of triplicate samples performed in a minimum of three independent experiments. For measuring cell elongation, a total of $\sim 75-100$ cells were analyzed from 9 images taken from three independent sample sets used for each condition. Comparisons between multiple groups were performed with single factor analysis of variance (ANOVA) followed by Tukey's post hoc test. Comparisons between two samples were performed with two-tailed Student's $t$-tests with post hoc analysis. Differences between two data sets were considered significant when $p<0.05$.

\section{Results and Discussion}

While the use of TEMED-polymerized PAA hydrogels is common practice, UV-polymerized PAA gels for use as cell substrates are now gaining momentum. Although TEMEDpolymerized gels offer a range of elastic moduli (Figure 1), 


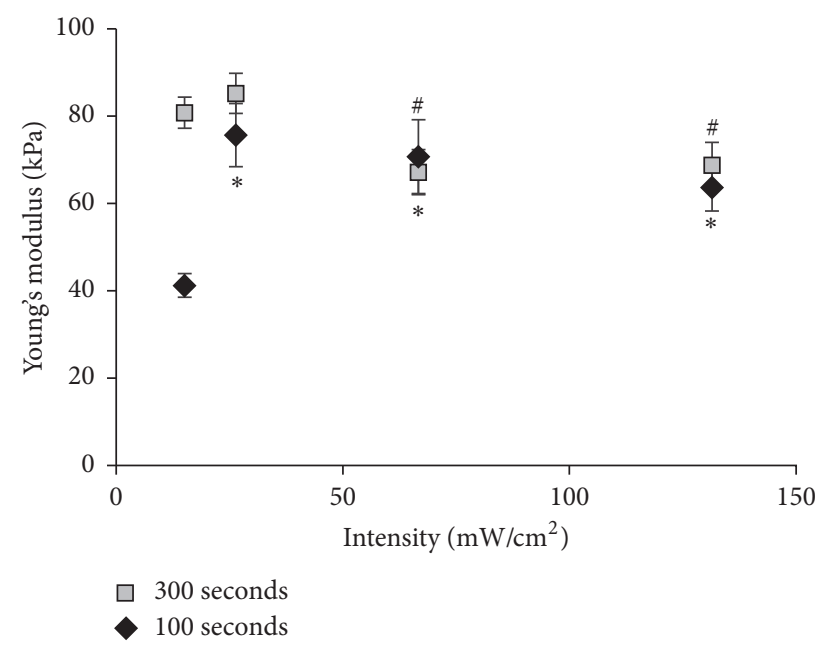

(a)

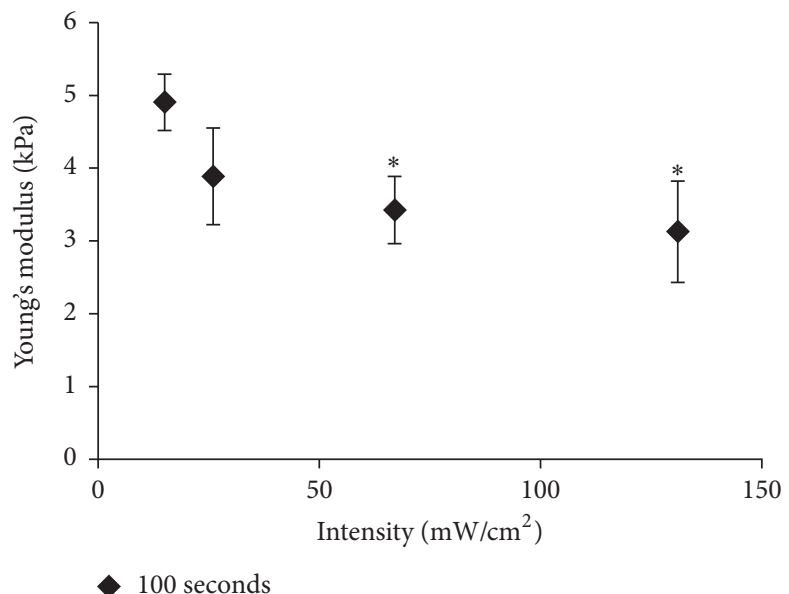

(b)

FIGURE 2: Young's modulus as a function of UV light intensity for (a) A12-B.25 and (b) A8-B.10, PAA hydrogels. Asterisks and pound symbols designate significant differences from Young's modulus for gels polymerized at $15 \mathrm{~mW} / \mathrm{cm}^{2}(p<0.05, n=3)$.

there are several distinct advantages to using UV polymerization. First, it allows for the formation of gradient gels which are excellent platforms for the investigation of cell motility, durotaxis, or differentiation $[1,10,12]$. Second, UV polymerization also allows for gel and surface patterning, providing a tool for evaluating cell behavior as a function of surface topography or composition [18, 19]. From a practical standpoint, UV crosslinking is faster; a typical gelation time for TEMED-based gels would be $\sim 30-45 \mathrm{~min}$, while the gelation time for UV-polymerized gels is $\sim 1-5$ min [1]. Lastly, the chemicals typically needed for UV polymerization (e.g., photoinitiators such as Irgacure) are less hazardous as opposed to the ones required for standard free radical polymerization, such as the toxic TEMED catalyst [8]. However, while both reactions are based on free radical polymerization, different parameters govern the polymerization kinetics and the resultant Young's modulus. Rather than trying to manipulate multiple parameters (UV exposure time and intensity) independently to obtain desired stiffness, we aimed to streamline the process, while simultaneously increasing reproducibility. Additionally, rather than using multiple gel compositions for subsequent experiments, we chose a soft hydrogel of lower acrylamide and bisacrylamide concentration (A8-B.10) and a stiff hydrogel of higher acrylamide and bisacrylamide concentration (A12-B.25). The A8-B.10 gel was chosen over the other soft hydrogels (A5-B.025 and A5-B.10), because it was easier to handle. Two different hydrogels were selected to ensure that the observed trends in modulus as a function of UV-polymerization parameters would hold for hydrogels of significantly different monomer and crosslinker concentration (i.e., resultant modulus).

3.1. Effect of UV Intensity. Here, we first examined the effect of light intensity on the final PAA hydrogel modulus(Figure 2). We specifically examined a soft (A8-B.10) and a stiff (A12-B.25) PAA hydrogel at two different exposure times: $100 \mathrm{~s}$ and $300 \mathrm{~s}$. We observed a bimodal dependence of gel modulus on UV light intensity. There was $\sim 83 \%$ increase in gel stiffness with a 74\% increase in light intensity (from 15 to $26 \mathrm{~mW} / \mathrm{cm}^{2}$ ) for the stiff gel at a short exposure time of $100 \mathrm{~s}$. However, rather than a further increase in modulus with an increase in intensity, we observed a slight, yet not statistically significant decrease in modulus. While not as significant, a similar trend was observed for the stiff gel at an exposure time of $300 \mathrm{~s}$. There was a slight, but insignificant, increase of $6 \%$ in Young's modulus with an increase in UV intensity from 15 to $26 \mathrm{~mW} / \mathrm{cm}^{2}$, followed by a statistically significant $20 \%$ decrease in modulus for the higher UV intensities of 67 and $131 \mathrm{~mW} / \mathrm{cm}^{2}$. For the softer A8-B.10 gel at an exposure time of $300 \mathrm{~s}$, the highest modulus was achieved at the lowest $15 \mathrm{~mW} / \mathrm{cm}^{2}$ UV light intensity; there was a $37 \%$ drop in modulus between gels made at $15 \mathrm{~mW} / \mathrm{cm}^{2}$ versus $131 \mathrm{~mW} / \mathrm{cm}^{2}$ intensity.

An increase in photopolymerized hydrogel modulus with increased light intensity was an anticipated result and can be explained by an improved crosslinking reaction and a greater number of functional crosslinks formed [20]. The second trend, which showed no further increase in modulus above a certain threshold UV light intensity, is not as well documented. For the free radical UV polymerization studied here, we postulate that the phenomena can be explained by overall faster polymerization kinetics and faster radical transfer, resulting in shorter polymer chains. Also, it is possible that complete photoinitiator consumption, leading to reaction completion past a threshold UV light intensity, can explain the lack of modulus increase. The rate of UV polymerization is directly proportional to the UV light intensity and photoinitiator concentration [21]. Thus, we note that, for a predetermined photoinitiator concentration, there was an optimal UV light intensity for achieving a higher modulus 


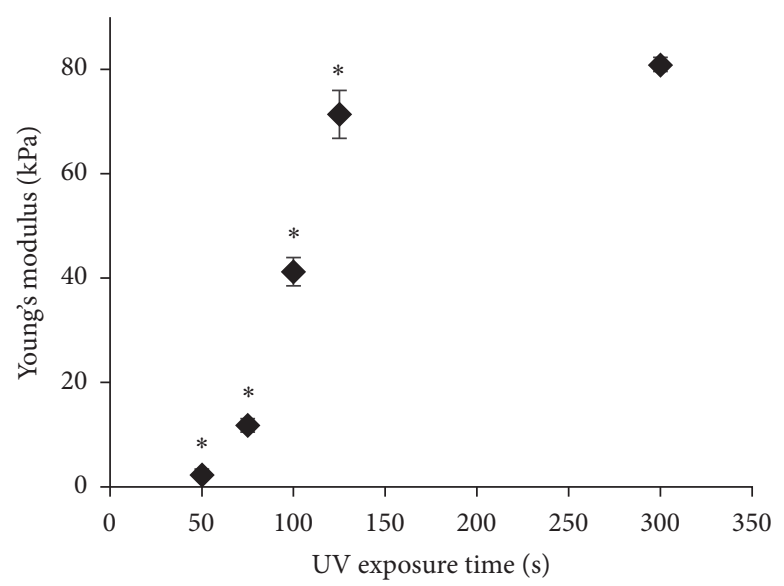

(a)

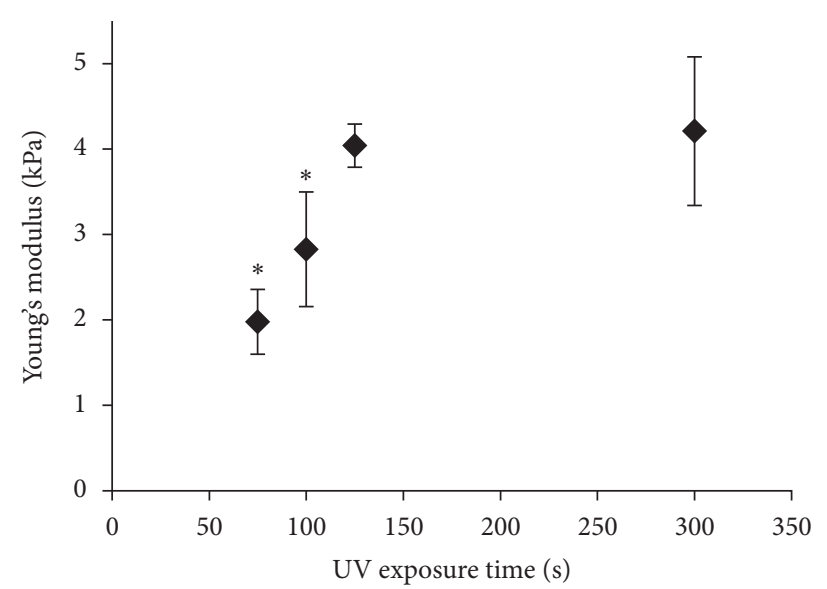

(b)

FIGURE 3: Young's modulus as a function of UV exposure time for (a) A12-B.25 and (b) A8-B.10, PAA hydrogels. Asterisks designate significant differences from Young's modulus for gels polymerized at an exposure time of $300 \mathrm{~s}(p<0.05, n=3)$.

from a given gel precursor solution, which was dependent on the total acrylamide and bisacrylamide concentration.

3.2. Effect of UV Exposure Time. For photopolymerized PAA hydrogels, UV exposure time has been utilized as the most effective strategy to achieve varying Young's moduli from the same hydrogel precursor solution $[1,10,12]$. As polymerization is a kinetic process, the longer exposure times allow for the reaction to proceed to completion. For this reason, longer polymerization times, up to a certain threshold, are expected to lead to a larger number of functional crosslinks, resulting in a higher Young's modulus. Further, it is possible that UV exposure time, by altering reaction rate, has an effect on the resultant hydrogel network defects, which have been shown to occur for PAA hydrogels, especially at higher acrylamide and bisacrylamide concentrations [22]. Increase in network defects (i.e., hydrogel heterogeneity) with an increase in reaction rate has been shown previously [21]. Network defects, on the other hand, have a pronounced effect on hydrogel modulus [23]. For example, chain entanglements cause an increase in crosslink density within the polymer network, which in turn results in a higher modulus $[24,25]$. Alternatively, intramolecular cycles (chain loops) decrease crosslink density, thus negatively affecting hydrogel modulus [21]. Furthermore, since the polymerization rate depends on the concentrations of the acrylamide monomer and bisacrylamide crosslinker, they are expected to change for each precursor solution. Here, we again tested a soft gel (A8-B.10) and a stiff gel (A12-B.25). As expected, the polymerization rate was dependent on the total acrylamide and bisacrylamide concentration (Figure 3).

We noted that longer polymerization times were needed to reach the gelation point for precursor solutions of lower total acrylamide and bisacrylamide concentration. For example, while only a $50 \mathrm{~s}$ exposure time was required to form the stiff gel (Figure 3(a)), a minimum exposure time of $75 \mathrm{~s}$ was needed to achieve gelation for the soft gel (Figure 3(b)). However, for both gels, we noted a significant increase in
Young's modulus for higher exposure times. For example, for the A12-B.25 gel, we saw a 97\% increase in Young's modulus for gels with an exposure time of $50 \mathrm{~s}$ versus gels with an exposure time of $300 \mathrm{~s}$. For the A8-B.10 gel, we saw a similarly large increase of $53 \%$ for gels with an exposure time of $75 \mathrm{~s}$ versus gels with an exposure time of $300 \mathrm{~s}$. Hence, by simply changing exposure time, we were able to achieve a large variation in stiffness in gels from the same gel precursor solution.

\subsection{Effect of Photoinitiator Concentration on PAA Hydrogel} Modulus. For UV-polymerized PAA gels, the photoinitiator Irgacure absorbs UV light to generate free radicals causing a chain reaction that leads to polymerization. The resulting polymer chain length affects the hydrogel modulus-the higher the chain length, the stiffer the resultant gel. One way to affect the polymer chain length is by altering the photoinitiator concentration. Increasing the photoinitiator concentration has been shown to result in multiple shorter polymer chains and hence a lower modulus [26]. Decreasing the photoinitiator concentration has been shown to result in a lower number, but higher molecular weight polymer chains leading to a higher modulus [26]. Our data corroborates previous findings: we observed a 1.3-fold increase in modulus when the Irgacure concentration was decreased from $0.5 \%$ to $0.1 \%$ and another 1.2 -fold increase in modulus when the Irgacure concentration was decreased from $0.1 \%$ to $0.01 \%$ (Figure 4).

3.4. Effect of $U V$ Dose. While above we demonstrated that both UV exposure time and UV light intensity could be used to control PAA hydrogel modulus, it is often impractical to test multiple samples to determine the optimal UV condition that would lead to an optimal hydrogel modulus. Along with manipulating intensity and time independently, we followed the evolution of hydrogel modulus as a function of total UV energy, that is, UV dose (see (1)). We again considered a soft gel (A8-B.10) and a stiff gel (A12-B.25). The data in Figure 5 


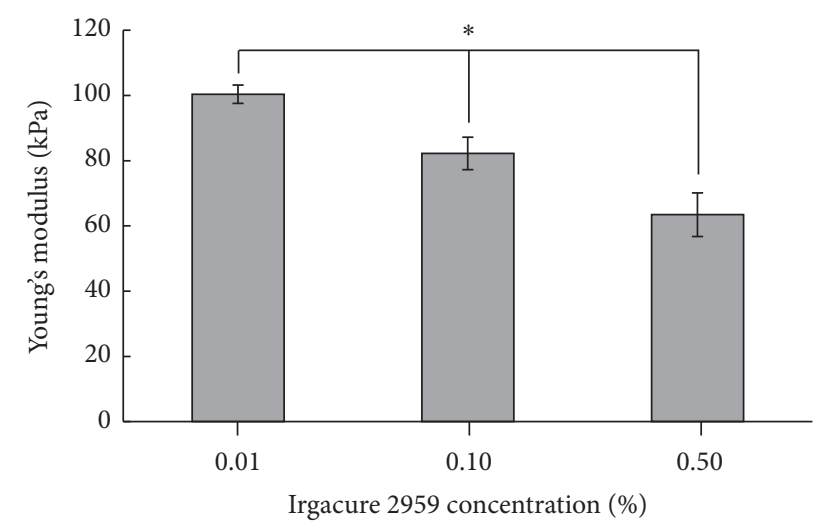

FIgURE 4: Young's modulus as a function of photoinitiator Irgacure 2959 concentration for A12-B.25 PAA hydrogel for a fixed UV intensity of $15 \mathrm{~mW} / \mathrm{cm}^{2}$ and fixed exposure time of $300 \mathrm{~s}$. Asterisks designate significant differences for $p<0.05, n=3$.

represents the change in Young's modulus as a function of UV dose, where dose was varied by a change in UV exposure time for a fixed light intensity. When presented in this fashion, we saw an important trend emerge: there was a threshold dose of $\sim 5 \times 10^{3} \mathrm{~mJ} / \mathrm{cm}^{2}$ at which no further increase in Young's modulus was observed for any hydrogel tested. It is important to note that this threshold value was achieved by manipulating either the UV intensity or the UV exposure time and was independent of the total acrylamide and bisacrylamide concentrations tested in this study. However, it is conceivable that using a significantly lower acrylamide or bisacrylamide concentration would affect the threshold modulus; the concentrations here were chosen to simulate the modulus range of most soft tissues in the body [3]. To the best of our knowledge, this trend has not been previously reported for UV-polymerized PAA hydrogels.

Furthermore, we saw a steep increase in Young's modulus for minimal changes in dose in the range of $0.8 \times 10^{3}$ to 5 $\times 10^{3} \mathrm{~mJ} / \mathrm{cm}^{2}$ and leveling off in the modulus for changes in dose in the range of $5 \times 10^{3}$ to $40 \times 10^{3} \mathrm{~mJ} / \mathrm{cm}^{2}$. Overall, the observed dose, as well as total time needed to fully polymerize the PAA hydrogel, aligned closely with data reported in the literature [12]. The above described trends along with the individual intensities and exposure times used are summarized in Table S1 (in the Supplementary Material available online at https://doi.org/10.1155/2017/5147482). Lastly, the moduli achieved via UV polymerization are similar to those measured when fabricating PAA hydrogels using traditional AP/TEMED polymerization (Figure 1). For example, for A12B. 25 UV-polymerized gel using UV intensity of $15 \mathrm{~mW} / \mathrm{cm} 2$ and $0.10 \%$ Irgacure concentration, Young's modulus was 93.8 $\pm 0.27 \mathrm{kPa}$, while the same gel made using AP/TEMED polymerization had Young's modulus of $112.3 \pm 8.0 \mathrm{kPa}$. It is important to note that further optimization of UVpolymerization conditions to achieve a higher modulus is possible. For example, decreasing the Irgacure concentration ten times (from $0.1 \%$ to $0.01 \% \mathrm{w} / \mathrm{v}$ ) increased Young's modulus of the A8-B.10 PAA and the A12-B.25 PAA gels, making them of the same modulus as the AP/TEMED-polymerized

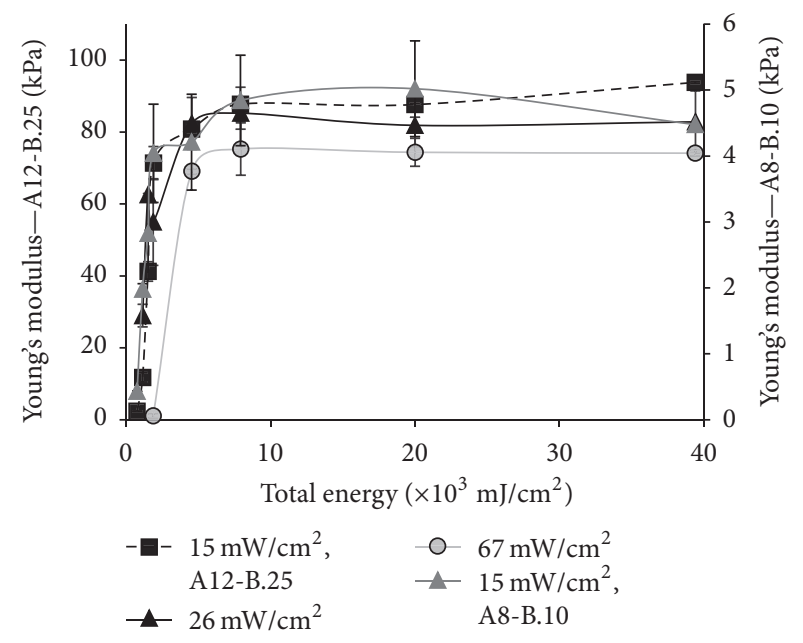

FIGURE 5: Young's modulus of two PAA hydrogel compositions as a function of UV dose calculated as UV intensity multiplied by UV exposure time.

hydrogels of the same composition (Table S2). Note that the exposure time was also increased for the lower Irgacure concentration to allow for complete polymerization to occur. This increase in modulus with the decrease in photoinitiator concentration could be attributed to higher molecular weight polymer chains (see Figure 4).

3.5. Cell Morphology on UV-Polymerized PAA Hydrogels. We also evaluated cell spreading on PAA hydrogels fabricated using UV polymerization. Our goal was to show that, regardless of the method with which the dose for PAA polymerization was obtained (i.e., high intensity but short exposure time versus low intensity by long exposure time), cell behavior would be identical on gels of identical stiffness. For this experiment, we maintained a constant dose of $8 \mathrm{~mJ} / \mathrm{cm}^{2}$ but altered the UV intensity and the exposure time. For one set of gels, the intensity was low $\left(15 \mathrm{~mW} / \mathrm{cm}^{2}\right)$ and the exposure time was high (524 s). Consequently, for another set of gels, the intensity was high $\left(67 \mathrm{~mW} / \mathrm{cm}^{2}\right)$ and the exposure time was low (119 s). We used tissue culture polystyrene (TCP) as control. We found that the cell spreading area on all three samples was $\sim 600 \mu \mathrm{m}^{2}$ and that there were no significant differences (Figure 6). This result corroborates the cell spreading area observed on the TEMED-polymerized gels of similar stiffness (Figure 7), indicating that UV polymerization can be utilized to fabricate PAA hydrogels for the use of substrates to assess cellular behavior.

\section{Conclusions}

In conclusion, we present a comprehensive analysis on the effect of various crosslinking parameters on the final PAA hydrogels' Young's modulus. Manipulating PAA hydrogels modulus is becoming increasingly important in the fields of basic cell biology, biophysics, tissue engineering, and regenerative medicine, where PAA gels are widely used as cell culture substrates. Importantly, for UV-polymerized hydrogels, we 
TCP $(2.5 \mathrm{GPa})$
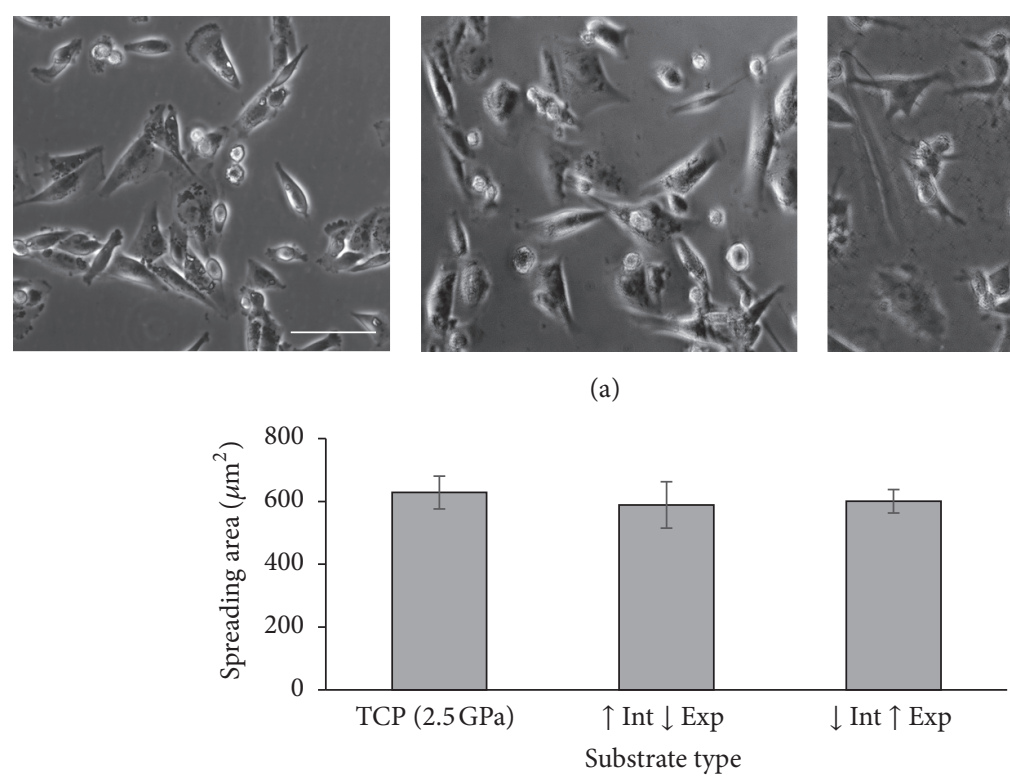

$\uparrow$ Int $\downarrow$ Exp

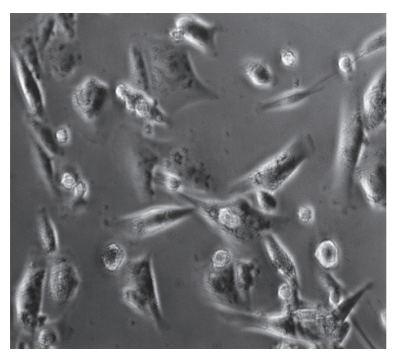

(a)

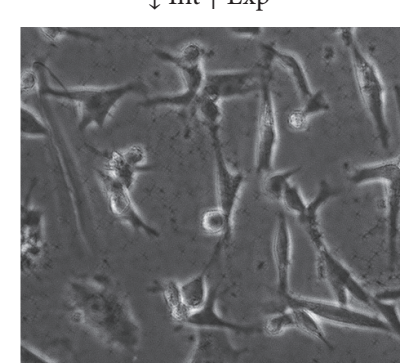

(b)

FIGURE 6: Cell morphology after $24 \mathrm{~h}$ of culture on collagen-coated UV-polymerized A12-B.25 PAA hydrogels. (a) Phase contrast images of MDA-MB-231 cells seeded onto PAA gels of equal dose, but with different exposure times and UV intensity. The " $\uparrow$ Int $\downarrow$ Exp" condition had an intensity of $67 \mathrm{~mW} / \mathrm{cm}^{2}$ and exposure time of $119 \mathrm{~s}$. The " $\downarrow$ Int $\uparrow$ Exp" condition had an intensity of $15 \mathrm{~mW} / \mathrm{cm}^{2}$ and exposure time of $524 \mathrm{~s}$. (b) No significant differences were found in the spreading area of MDA-MB-231 cells seeded onto PAA gels of equal dose $\left(8 \mathrm{~mJ} / \mathrm{cm}^{2}\right)$, but with different exposure time and UV intensity (same as in (a)). Scale bar represents $100 \mu \mathrm{m}$.
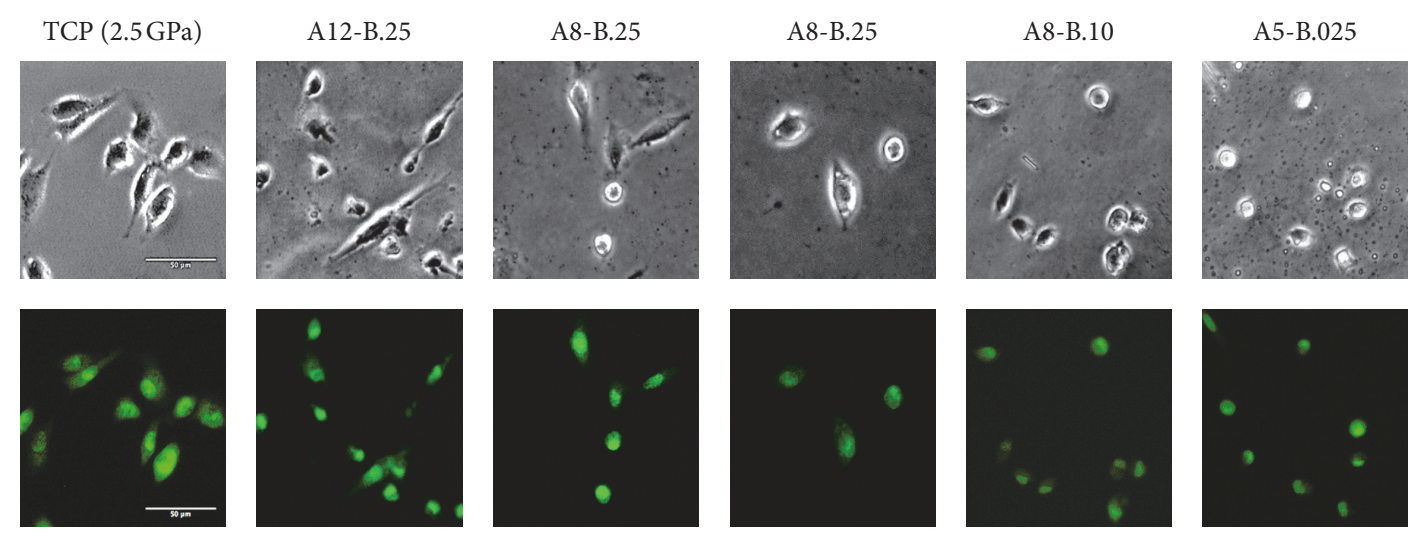

(a)

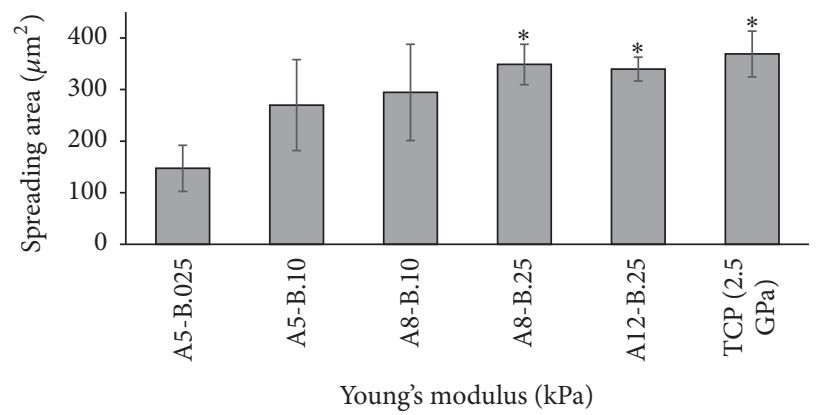

(b)

Figure 7: Cell morphology after $24 \mathrm{~h}$ of culture on collagen-coated PAA gels of varying stiffness. (a) Phase contrast (upper panel) and fluorescent (lower panel, acridine orange staining) images of MDA-MB-231 cells seeded on PAA gels of different stiffness. (b) Spreading area of MDA-MB-231 cells seeded on PAA gels of different stiffness. Asterisks designate significant differences from the $1 \mathrm{kPa}$ hydrogel $(p<0.05$, $n=3)$. 
demonstrated that, rather than tuning the hydrogel modulus via UV intensity or exposure time independently, one should consider the UV dose for the system. For our system, which utilized $0.1 \% \mathrm{w} / \mathrm{v}$ of Irgacure photoinitiator, the threshold dose was found to be $5 \times 10^{3} \mathrm{~mJ} / \mathrm{cm}^{2}$; below this energy, we saw an abrupt increase in compliance for incremental changes in energy, while we did not observe any significant changes in hydrogel compliance for any changes in UV dose above the threshold value. The cell spreading observed in PAA gels prepared using UV polymerization corroborated results seen in the TEMED-polymerized PAA gels. Our comprehensive analysis on the effect of implementing UV dose could serve as a useful guide to increase the reproducibility and ease of use for the fabrication of UV-polymerized PAA hydrogels for many research applications.

\section{Disclosure}

Hunter Stevenson's current affiliation is Department of Bioengineering, University of Texas at Dallas, 2850 Rutford Ave., Richardson, TX 75080, USA. Era Jain's current affiliation is Department of Biomedical Engineering, Washington University in St. Louis, 6201 Forsyth Blvd., St. Louis, MO 63105, USA.

\section{Conflicts of Interest}

The authors declare that there are no conflicts of interest regarding this paper.

\section{Acknowledgments}

Funding was provided by start-up funds awarded to Dr. Silviya Zustiak from Saint Louis University.

\section{References}

[1] J. R. Tse and A. J. Engler, Current Protocols in Cell Biology, 2010, 10.16. 11-10.16. 16.

[2] R. J. Pelham Jr. and Y.-L. Wang, "Cell locomotion and focal adhesions are regulated by substrate flexibility," Proceedings of the National Academy of Sciences of the United States of America, vol. 94, no. 25, pp. 13661-13665, 1997.

[3] I. Levental, P. C. Georges, and P. A. Janmey, "Soft biological materials and their impact on cell function," Soft Matter, vol. 3, no. 3, pp. 299-306, 2007.

[4] C.-M. Lo, H.-B. Wang, M. Dembo, and Y.-L. Wang, "Cell movement is guided by the rigidity of the substrate," Biophysical Journal, vol. 79, no. 1, pp. 144-152, 2000.

[5] T. Yeung, P. C. Georges, L. A. Flanagan et al., "Effects of substrate stiffness on cell morphology, cytoskeletal structure, and adhesion," Cell Motility and the Cytoskeleton, vol. 60, no. 1, pp. 24-34, 2005.

[6] R. W. Tilghman, C. R. Cowan, J. D. Mih et al., "Matrix rigidity regulates cancer cell growth and cellular phenotype," PLOS ONE, vol. 5, no. 9, Article ID e12905, 2010.

[7] R. W. Tilghman, E. M. Blais, C. R. Cowan et al., "Matrix rigidity regulates cancer cell growth by modulating cellular metabolism and protein synthesis," PLoS ONE, vol. 7, no. 5, Article ID e37231, 2012.
[8] S. Zustiak, R. Nossal, and D. L. Sackett, "Multiwell stiffness assay for the study of cell responsiveness to cytotoxic drugs," Biotechnology and Bioengineering, vol. 111, no. 2, pp. 396-403, 2014.

[9] J. D. Mih, A. S. Sharif, F. Liu, A. Marinkovic, M. M. Symer, and D. J. Tschumperlin, "A multiwell platform for studying stiffnessdependent cell biology," PLoS ONE, vol. 6, no. 5, Article ID e19929, 2011.

[10] A. M. Kloxin, J. A. Benton, and K. S. Anseth, "In situ elasticity modulation with dynamic substrates to direct cell phenotype," Biomaterials, vol. 31, no. 1, pp. 1-8, 2010.

[11] Y. Aratyn-Schaus, P. W. Oakes, J. Stricker, S. P. Winter, and M. L. Gardel, "Preparation of Complaint Matrices for Quantifying Cellular Contraction," Journal of Visualized Experiments, no. 46, 2010.

[12] R. Sunyer, A. J. Jin, R. Nossal, and D. L. Sackett, "Fabrication of Hydrogels with Steep Stiffness Gradients for Studying Cell Mechanical Response," PLoS ONE, vol. 7, no. 10, Article ID e46107, 2012.

[13] C. G. Williams, A. N. Malik, T. K. Kim, P. N. Manson, and J. H. Elisseeff, "Variable cytocompatibility of six cell lines with photoinitiators used for polymerizing hydrogels and cell encapsulation," Biomaterials, vol. 26, no. 11, pp. 1211-1218, 2005.

[14] B. K. Mann, A. S. Gobin, A. T. Tsai, R. H. Schmedlen, and J. L. West, "Smooth muscle cell growth in photopolymerized hydrogels with cell adhesive and proteolytically degradable domains: Synthetic ECM analogs for tissue engineering," Biomaterials, vol. 22, no. 22, pp. 3045-3051, 2001.

[15] S. P. Zustiak and J. B. Leach, "Hydrolytically degradable poly(ethylene glycol) hydrogel scaffolds with tunable degradation and mechanical properties," Biomacromolecules, vol. 11, no. 5, pp. 1348-1357, 2010.

[16] T. Boudou, J. Ohayon, C. Picart, and P. Tracqui, "An extended relationship for the characterization of Young's modulus and Poisson's ratio of tunable polyacrylamide gels," Biorheology, vol. 43, no. 6, pp. 721-728, 2006.

[17] S. Syed, A. Karadaghy, and S. Zustiak, "Simple polyacrylamidebased multiwell stiffness assay for the study of stiffnessdependent cell responses," Journal of Visualized Experiments, vol. 2015, no. 97, Article ID e52643, 2015.

[18] M. J. Poellmann, P. A. Harrell, W. P. King, and A. J. W. Johnson, "Geometric microenvironment directs cell morphology on topographically patterned hydrogel substrates," Acta Biomaterialia, vol. 6, no. 9, pp. 3514-3523, 2010.

[19] H. Shin, "Fabrication methods of an engineered microenvironment for analysis of cell-biomaterial interactions," Biomaterials, vol. 28, no. 2, pp. 126-133, 2007.

[20] S. Seiffert, W. Oppermann, and K. Saalwächter, "Hydrogel formation by photocrosslinking of dimethylmaleimide functionalized polyacrylamide," Polymer, vol. 48 , no. 19 , pp. 55995611, 2007.

[21] B. D. Fairbanks, M. P. Schwartz, C. N. Bowman, and K. S. Anseth, "Photoinitiated polymerization of PEG-diacrylate with lithium phenyl-2,4,6-trimethylbenzoylphosphinate: polymerization rate and cytocompatibility," Biomaterials, vol. 30, no. 35, pp. 6702-6707, 2009.

[22] J. Baselga, M. A. Llorente, I. Hernández-Fuentes, and I. F. Piérola, "Network defects in polyacrylamide gels," European Polymer Journal, vol. 25, no. 5, pp. 471-475, 1989.

[23] A. S. Hoffman, "Hydrogels for biomedical applications," Advanced Drug Delivery Reviews, vol. 64, pp. 18-23, 2012. 
[24] W. Chassé, M. Lang, J. Sommer, and K. Saalwächter, "CrossLink Density Estimation of PDMS Networks with Precise Consideration of Networks Defects," Macromolecules, vol. 45, no. 2, pp. 899-912, 2012.

[25] O. Okay and S. Durmaz, "Charge density dependence of elastic modulus of strong polyelectrolyte hydrogels," Polymer, vol. 43, no. 4, pp. 1215-1221, 2001.

[26] N. S. Allen, J. Segurola, M. Edge, E. Santamari, A. McMahon, and S. Wilson, "A comparative kinetic study of commercial photoinitiators for UV/visible curable acrylate clear coatings," Surface Coatings International Part B: Coatings International, vol. 82, no. 2, pp. 67-76, 1999. 

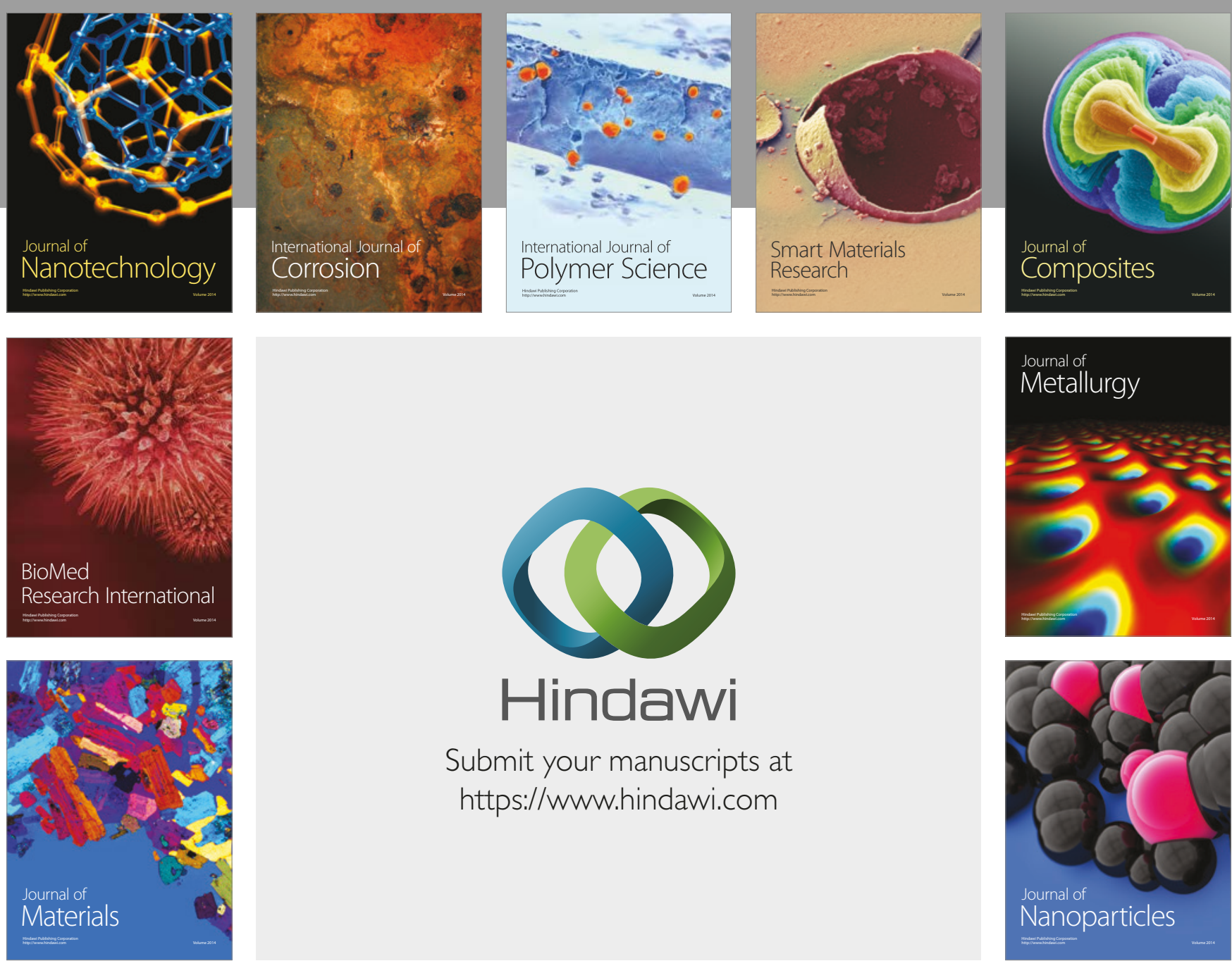

\section{Hindawi}

Submit your manuscripts at

https://www.hindawi.com
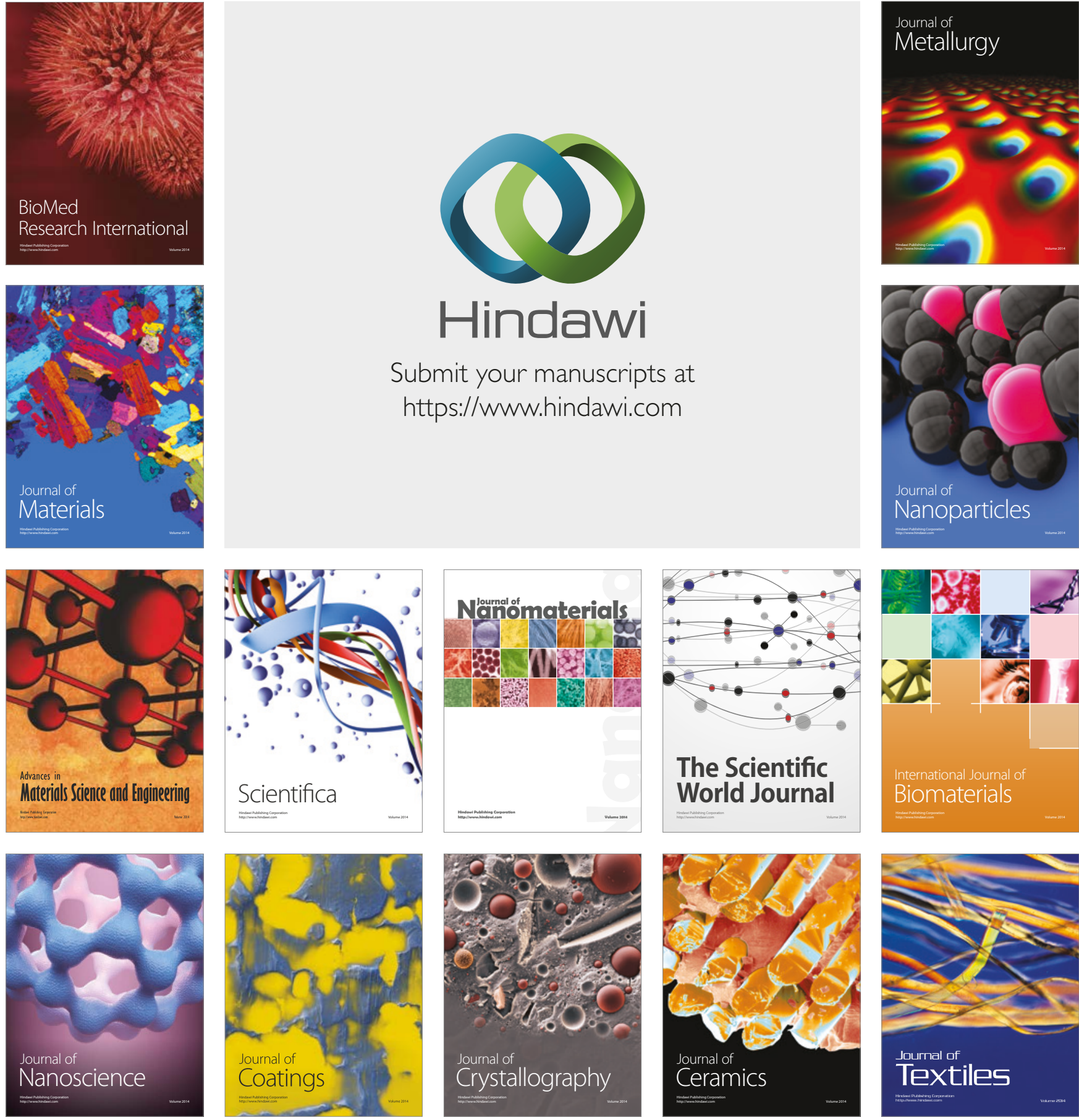

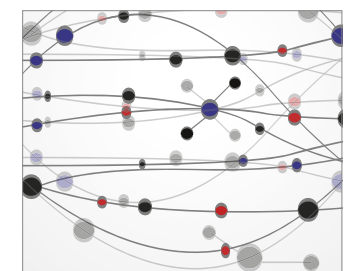

The Scientific World Journal
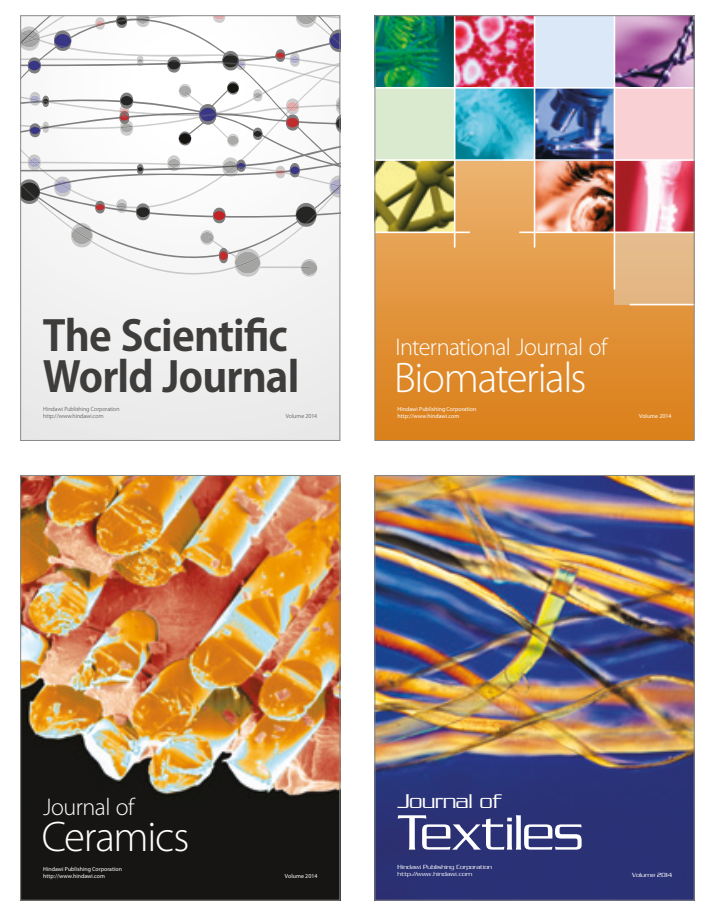